\title{
Le lichen plan dans la réaction du greffon contre l'hôte
}

\section{Lichen planus and Graft-versus-Host Disease}

\author{
SYLVIE BOISRAME-GASTRIN ${ }^{1}$, FRANCIS PASCAL², ELIANE GLUCKMANN ${ }^{3}$, \\ ALAIN DEBOISE², CHRISTIAN BERTHOU ${ }^{4}$, FRANÇOIS PREDINE-HUG'
}

\section{RÉSUMÉ}

La réaction du greffon contre l'hôte (GVHD pour Graft-versus-Host Disease) touche de nombreux tissus ou organes. Cette maladie intéresse la sphère bucco-dentaire et comporte des manifestations muqueuses, salivaires et dentaires. Une étude préliminaire réalisée conjointement avec les équipes médicales de l'hôpital Saint Louis (Paris) et de l'hôpital Morvan (Brest) a permis d'étudier le lichen plan buccal dans la GVHD chez 36 patients. La transformation maligne du lichen dans la GVHD est contestée dans la littérature, mais elle est bien présente et elle survient longtemps après l'allogreffe de moelle osseuse. Une prise en charge odontostomatologique permet de suivre l'évolution des lésions lichéniennes, de réduire les effets secondaires de I'hyposialie et de dépister les éventuelles transformations malignes tardives. (Med Buccale Chir Buccale 2006 ; 12: 47-56).

mots clés : réaction du greffon contre hôte, lichen plan, carcinome épidermoïde

médecine buccale chirurgie buccale

VOL. $12, \mathrm{~N}^{\circ} 1$ 2006

page 47

\section{SUMMARY}

The Graft-versus-Host Disease (GVHD) touches many tissues or bodies. This disease interests the oral cavity and includes mucous, salivary and dental demonstrations. A preliminary study carried out jointly with the medical teams of Saint Louis hospital (Paris) and Morvan hospital (Brest) made it possible to study the oral plane lichen in the GVHD among 36 patients. The malignant transformation of the lichen in the GVHD is disputed in the literature, but it is quite present and it occurs a long time after the allogenic bone marrow transplantation.

An odonto-stomatologic assumption of responsibility makes it possible to follow the evolution of the lichen planus lesions, to reduce the side effects of hyposialy, and to detect possible the late malignant transformations. (Med Buccale Chir Buccale 2006; 12: 47-56).

key words: graft-versus host disease, lichen planus, squamous cell carcinoma

1- Service d'Odontologie - C.H.U. Morvan - Brest

2- Service de Stomatologie - C.H.U. Saint Louis - Paris

3- Service d'Hématologie - C.H.U. Saint Louis - Paris

4- Service d'Hématologie - C.H.U. Morvan - Brest

Demande de tirés à part :

Sylvie Boisramé-Gastrin Service d'Odontologie CHU Morvan 2 rue A Le Faux 29200 Brest France sylvieboisrame@hotmail.com 
médecine

buccale

chirurgie

buccale

VOL. $12, \mathrm{~N}^{\circ} 1$ 2006

page 48
La réaction du greffon contre l'hôte, ou GVHD pour Graft-versus-Host Disease, est une complication des greffes de moelle osseuse. Elle est due à la réponse de cellules immunocompétentes d'un donneur histocompatible contre les tissus du receveur. Essentiellement observée dans les allogreffes, on la rencontre également quand des lymphocytes, même en petit nombre, sont transférés à un organisme immunodéprimé. Dans sa forme aiguë (GVHDa) ou chronique (GVHDc), elle touche classiquement trois organes cibles qui sont respectivement la peau, en réalité le revêtement cutanéo-muqueux, le foie et le tube digestif, pour ensuite s'étendre à tout l'organisme.

L'incidence des lésions buccales en période aiguë varie de 25 à $75 \%$ [1,2]. Dans la forme chronique, l'atteinte buccale est la manifestation la plus communément observée chez les sujets allogreffés. Elle représente parfois la première, voire la seule expression, de la phase chronique de la maladie, touchant $80 \%$ des patients [3]. Les manifestations les plus fréquentes sont le lichen plan associé à un syndrome sec ; ce dernier étant à l'origine de mucocèles, polycaries et de parodontopathies. Egalement liées à l'immunodépression chronique, ces manifestations, quelquefois torpides, favorisent les surinfections secondaires bactérienne, mycosique et virale.

Les lésions cutanéo-muqueuses buccales sont donc difficiles à gérer, tant pour l'équipe soignante que pour le patient. De plus, la transformation éventuelle des lésions lichéniennes peut apparaître longtemps après l'allogreffe. II a donc paru intéressant de détailler les lésions buccales chez les patients atteints de GVHD, de préciser leur localisation et d'analyser l'évolution de certaines lésions.

Cette étude a pour buts de :

- répertorier les différents types de lésions endobuccales des GVHDc et leur siège,

- suivre l'évolution de ces lésions sur les plans clinique et anatomopathologique,

- déterminer les sièges préférentiels de ces lésions en voie de transformation maligne,

- préciser les éléments prédictifs cliniques et anatomopathologiques de la transformation maligne pour tenter un traitement préventif, voire curatif, du carcinome épidermoïde à un stade précoce.

\section{MATERIEL ET METHODES}

Cette étude a eu pour cadre le Service de stomatologie du Centre hospitalo-universitaire de I'hôpital Saint-Louis à Paris et le Service d'odontologie du Centre hospitalo-universitaire de l'hôpital Morvan à Brest. Les patients de cette étude sont avant tout suivis dans un service d'hématologie. L'échantillon de l'étude est composé de 36 patients âgés de 11 à 57 ans.

Un tableau récapitulatif a été élaboré en utilisant les données des dossiers médicaux. II comporte les éléments suivants : l'âge, la situation familiale, les addictions (intoxications alcoolique, tabagique ou autres), les antécédents médicaux à savoir l'affection hématologique initiale, la date de la découverte de l'affection, le type de greffe de moelle, le sexe du donneur, le protocole thérapeutique : (chimio et/ou radiothérapie), la date de début de la GVHD, la présence ou non d'un syndrome sec, le siège, la date d'apparition et le type des lésions lichéniennes, et enfin la transformation maligne avec le siège et le type de la tumeur.

Les méthodes statistiques utilisées pour cette étude sont le test de khi-2 et le test exact de Fisher pour les comparaisons de proportion. La distribution des variables descriptives a également été explorée par méthodes graphiques. Pour tous les tests effectués, le seuil de signification préalablement choisi est $\alpha=0,05$.

\section{RESULTATS}

L'échantillon est composé de 18 hommes et 18 femmes, dont 18 sont célibataires, 14 sont mariés ou vivant en concubinage, 4 divorcés. Concernant l'intoxication éthylique et/ou tabagique, seuls six patients de l'échantillon consomment régulièrement du tabac et de l'alcool. Les affections hématologiques initiales ayant conduit à une allogreffe de moelle osseuse sont résumés dans la Fig. 1. Il s'agit principalement des leucémies myéloïdes chroniques (30\% des cas) et des leucémies aigues lymphoblastiques (LAL) (25\% des cas).

Tous les patients ont reçu un conditionnement pré-greffe qui comporte une poly-chimiothérapie 


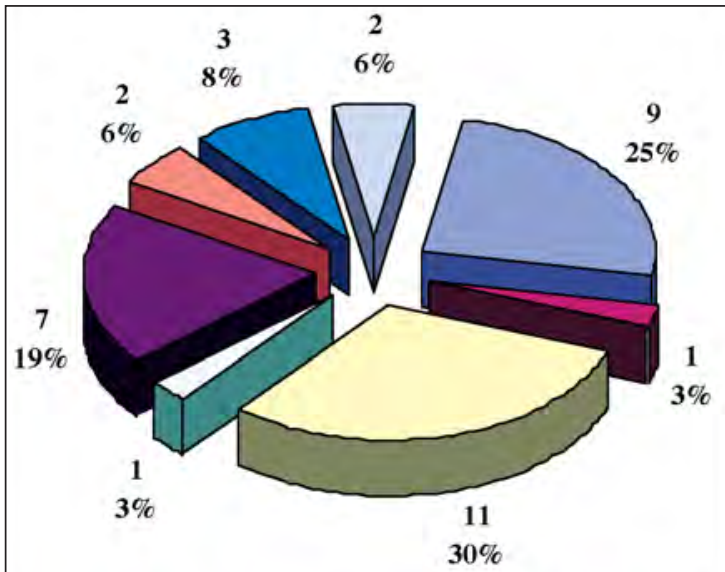

$\square \mathrm{LAL} \square \mathrm{LAM} \square \mathrm{LMC} \square \mathrm{MH}$ घAF $\square \mathrm{TG} \square \mathrm{AM} \square \mathrm{LNH}$

\section{Légende:}

LAL : leucémie aiguë lymphoblastique (acute lymphoblastic leukaemia)

MH : maladie de Hodgkin (Hodgkin's dlsease) LAM : leucémie aiguë myéloblastique (acute myelobastic leukaemia)

AF : anémie de Fanconi (Fanconi anemia)

LMC : leucémie myéloïde chronique (chronic myeloid leukaemia)

AM : aplasie médullaire (medullary aplasia)

TG : thrombasthénie de Glanzmann

(Glanzmann thrombasthenia)

LNH : lymphome non hodgkinien (non

Hodgkin's lymphoma)
Figure 1 : Affection hématologique initiale

Initial hematologic pathology

associée, à une irradiation corporelle totale, pour 24 d'entre eux (66,6 \%).

L'allogreffe de moelle osseuse est réalisée à partir d'un donneur familial de la fratrie ou d'un donneur anonyme : 15 donneurs sont de sexe masculin (13 de fratrie et 2 anonymes) et 21 de sexe féminin (19 de fratrie et 2 anonymes). Dans cet échantillon, la majorité des allogreffes de moelle osseuse est donc réalisée à partir de membres de la fratrie (Fig. 2). L'âge moyen des patients receveurs au moment de la greffe est de 25,6 ans. Le délai moyen d'apparition de la GVHDc après allogreffe de moelle osseuse est de 149,79 jours ; cette variable ne peut être retenue étant donnée l'absence de données pour 7 patients.

Les patients présentant un lichen lors d'une GVHD développent conjointement plusieurs formes cliniques. II y a trois formes prédominantes : la forme réticulée (23\%), la forme érosive (20\%) et la forme atrophique (20 \%) (Fig. 3).

Dans la GVHD, le lichen siège, par ordre de fréquence décroissant, sur la face interne des joues, la face dorsale de langue et à égalité dans le vestibule et sur le palais (Fig. 4). En associant les faces ventrale, dorsale et les bords latéraux de la langue, on arrive à un pourcentage de $30 \%$. La langue représente le siège principal de développement du lichen dans cet échantillon.

Le lichen a ensuite été comparé deux à deux à d'autres variables à l'aide du test de Fisher exact.

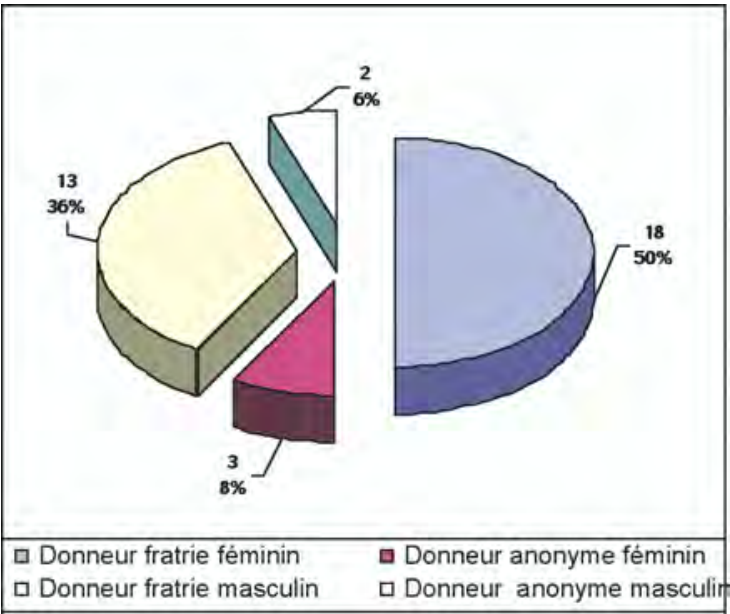

Figure 2 : Effectif des donneurs de moelle osseuse Bone marrow donors

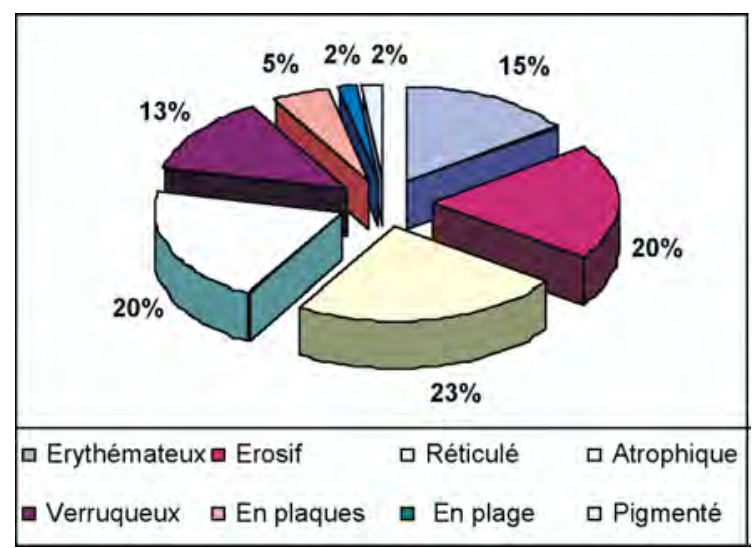

Figure 3 : Les différentes formes cliniques de lichen dans la GVHD

Various clinical shapes of lichen in GVHD médecine

buccale

chirurgie

buccale

VOL. $12, \mathrm{~N}^{\circ} 1$ 2006

page 49 


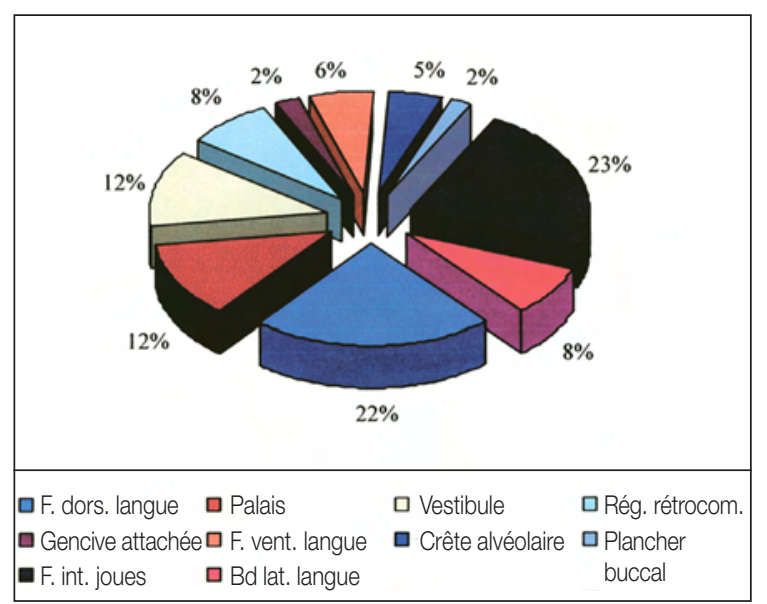

Figure 4 : Différentes localisations du lichen dans la GVHD

Various localizations of lichen in GVHD

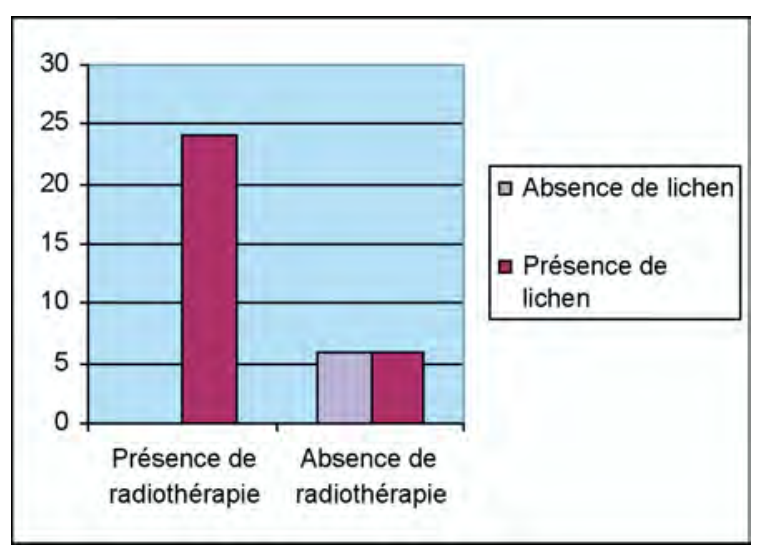

Figure 5 : Relation entre le lichen dans la GVHD et le protocole thérapeutique

Relation between oral lichen planus in GVHD and therapeutic protocol

Malheureusement les résultats sont peu significatifs. Ce manque de significativité peut être expliqué par le manque de puissance de notre échantillon. Cependant, certains résultats sont comparables à ceux retrouvés dans la littérature. Il semble judicieux de rechercher l'impact du conditionnement pré-greffe sur l'apparition du lichen. Il est difficile d'évaluer le rôle de la chi-

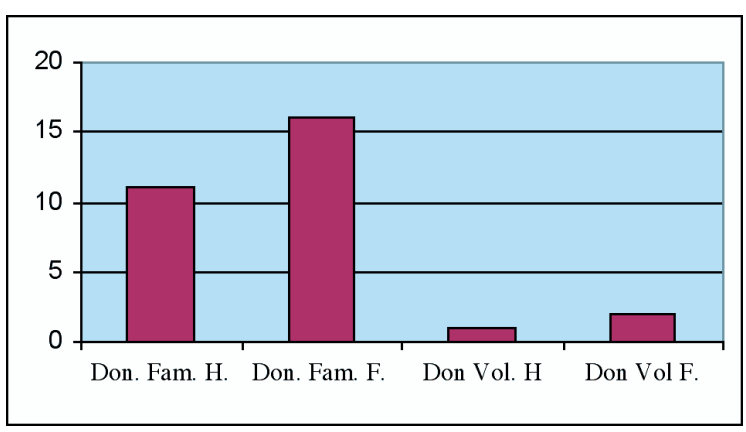

Figure 6 : Relation entre le lichen dans la GVHD et le type d'allogreffe

Relation between oral lichen planus in GVHD and allogeneic bone marrow transplantation

miothérapie (absence de données) ; mais $80 \%$ des patients ayant eu une irradiation corporelle totale développent par la suite un lichen (Fig. 5). Cette analyse statistique retrouve une significativité de ces deux variables. Le test exact de Fisher est ici $\mathrm{p}<10^{-4}$.

Si l'on prend en compte le type d'allogreffe effectué, les femmes donneuses ont tendance à provoquer plus de lichen chez les sujets allogreffés : $60 \%$ des patients présentant un lichen ont bénéficié d'une allogreffe de moelle osseuse provenant d'une femme (Fig. 6).

Cinq sujets allogreffés sur 36 ont présenté un carcinome épidermoïde buccal (13,88 \%). La transformation maligne s'est développée sur un lichen chronique. Ces quelques cas de transformation maligne ne suffisent pas à émettre des conclusions significatives.

Les cinq cas de transformation maligne sont survenus après les affections hématologiques suivantes : leucémie myéloïde chronique (1 cas), anémie de Fanconi (2 cas), maladie de Hodgkin (1 cas) et aplasie médullaire (1 cas). Le délai entre l'allogreffe de moelle osseuse et le développement d'un carcinome épidermoïde est en moyenne de 175 mois (Tab. 1), soit un délai d'apparition de 14,58 ans.

Tableau 1 : Délai d'apparition du carcinome épidermoïde buccal Time of appearance of oral squamous cell carcinoma

\begin{tabular}{|c|c|c|c|c|}
\hline LMC & A Fanconi 1 & A Fanconi 2 & Mal Hodgkin & Aplasie Med \\
\hline 186 mois soit & 113 mois soit & 259 mois soit & 130 mois soit & 187 mois soit \\
15,5 ans & 9,4 ans & 21,5 ans & 10,8 ans & 15,5 ans \\
\hline
\end{tabular}


La langue est dans $60 \%$ des cas le siège du carcinome épidermoïde (Tab. 2).

Tableau 2 : Siège des carcinomes épidermoïdes observés Site of oral squamous cell carcinoma observed

\begin{tabular}{|c|c|c|c|c|}
\hline LMC & A Fanconi 1 & A Fanconi 2 & Mal Hodgkin & Aplasie Med \\
\hline $\begin{array}{c}\text { Bord latéral } \\
\text { langue }\end{array}$ & $\begin{array}{c}\text { Vestibule inf. } \\
\text { droit }\end{array}$ & $\begin{array}{c}\text { Bord latéral } \\
\text { langue }\end{array}$ & $\begin{array}{c}\text { Face dorsale } \\
\text { langue }\end{array}$ & Palais \\
\hline
\end{tabular}

\section{DISCUSSION}

Dans la GVHD, le lichen plan représente une dermatose inflammatoire chronique, fréquente, qui comporte des localisations cutanées et muqueuses ; ces dernières étant les plus préoccupantes. Il est retrouvé quasi systématiquement dans les différentes poussées de GVHD et marque, selon certains auteurs, le début de la phase chronique de la GVHD, siégeant sur les zones les plus atteintes pendant la phase aiguë [4]. La pathogénie du lichen plan est immunologique et il est considéré comme faisant partie du spectre de la GVHD [5]. Dans la GVHDc, des lésions lichéniennes apparaissent de façon précoce mais les lésions de type atrophique se développent plus tardivement. Le lichen dans la GVHD résulte d'un processus alloimmun. Ce processus doit être très surveillé en raison du risque important chez ces patients de développer un carcinome secondaire.

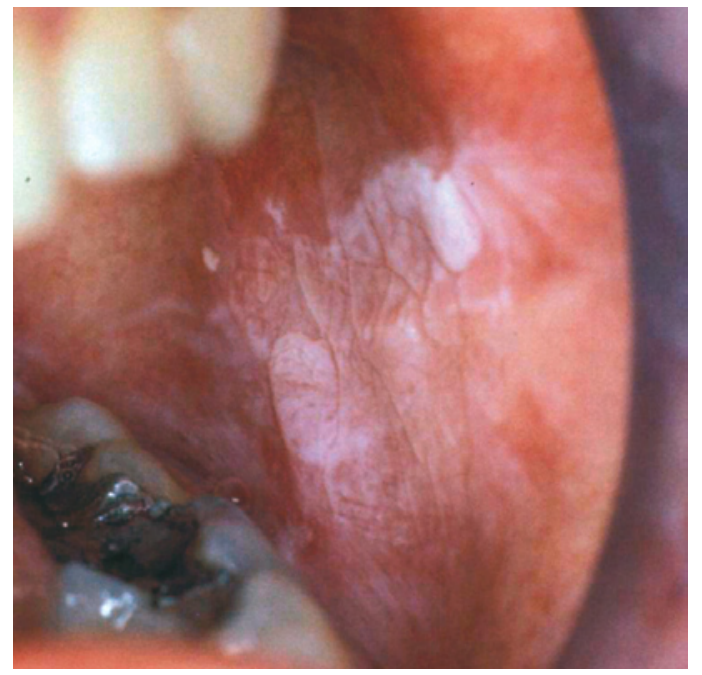

Figure 7 : Lichen réticulé dans la GVHD sur la joue gauche.

Reticulated lichen of GVHD on the left cheek
La forme réticulée se traduit par un réseau blanchâtre siégeant préférentiellement sur les joues (face postéro-interne) (Fig. 7), les gencives, la langue, le palais et les lèvres (Fig. 8). Asymptomatiques, les lésions réticulées récentes sont constituées de pointillés confluant en nappes ou plaques. La forme érosive (Fig. 9 et 10) se traduit par des lésions douloureuses, à limites nettes, recouvertes d'un enduit fibrino-leucocytaire (pseudomembrane), entourées d'un érythème, accompagnées ou non de lésions réticulées. La forme atrophique (Fig. 11) succède le plus souvent à un lichen érosif ou aux érosions développées lors de la GVHDa.

Un tableau comparatif entre le lichen plan idiopathique et le lichen plan dans la GVHDc est proposé (Tab. 3). Il est important de noter les principales différences du lichen plan dans la GVHDc : peu de lésions réticulées, nombreuses lésions kératosiques en plaques et verruqueuses,

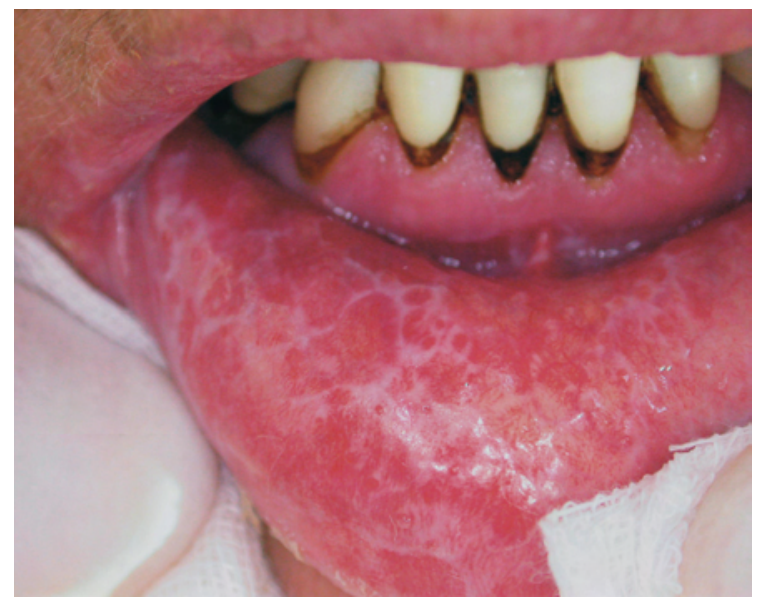

Figure 8 : Lichen réticulé dans la GVHD sur le versant muqueux labial inférieur.

Reticulated lichen of GVHD on the lower labial mucous slope médecine

buccale

chirurgie

buccale

VOL. $12, N^{\circ} 1$ 2006

page 51 


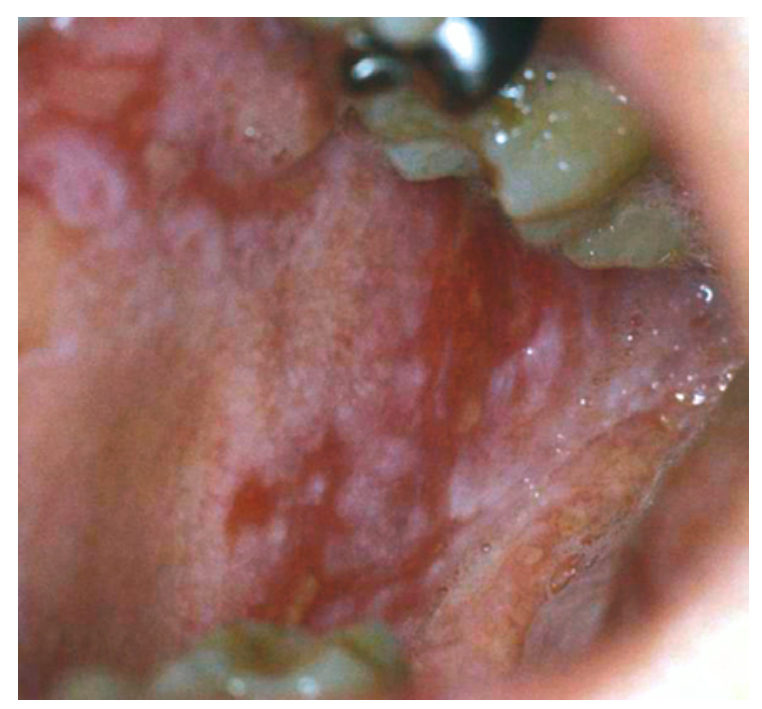

Figure 9 : Présence d'érosions lichéniennes entourées d'un érythème sur une nappe lichénienne jugale droite. Presence of lichen's erosions surrounded of an erythema on a right tablecloth cheek

médecine

buccale

chirurgie

buccale

VOL. $12, \mathrm{~N}^{\circ} 1$ 2006

page 52

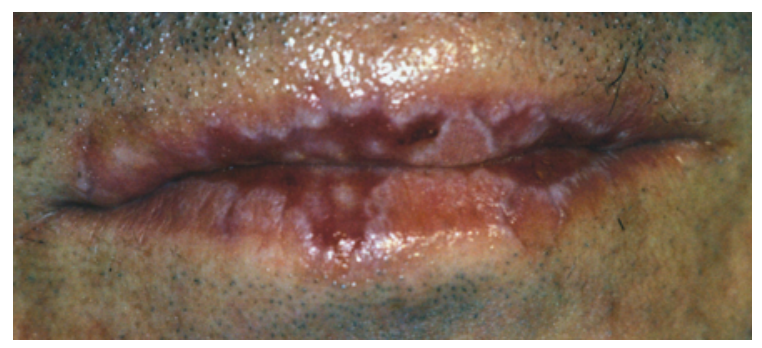

Figure 10 : Nouvelle poussée de GVHDc buccale avec présence d'érosions lichéniennes sur la demi-muqueuse labiale.

New oral push of GVHDc with presence of lichen erosions on the labial half-mucous membran

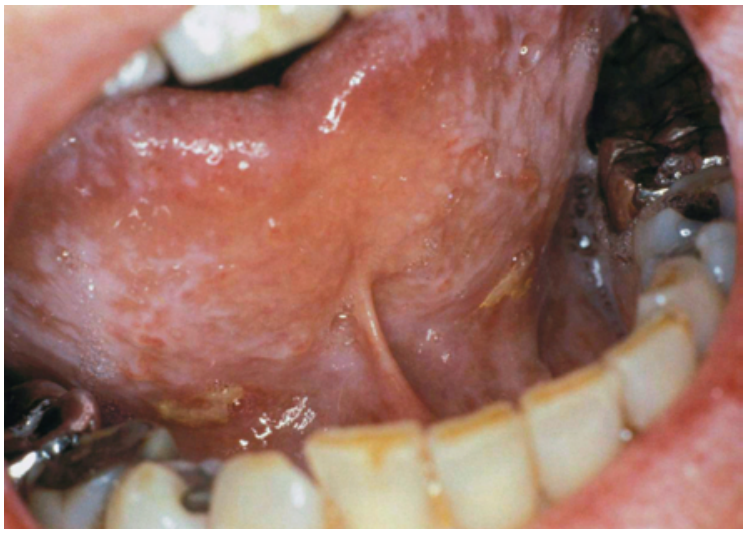

Figure 11 : Plages de lichen dans la GVHD sur la face ventrale de la langue avec deux érosions dans le sillon pelvi-lingual.

Beaches of lichen of GVHD on the ventral face of the tongue with two erosions in the pelvi-lingual furrow

érosions torpides fréquentes, atrophie constante et forte propension à la transformation maligne. La physiopathologie du lichen n'est pas encore élucidée. Elle est associée à un mécanisme immunologique mettant en jeu des lymphocytes $T$ et des allèles particuliers du système HLA comme HLA A 3,5,28, HLA-B16, w35,27,51, w57, HLADR1,2,3,w9,9 et HLA-DQW1 [6].

Histologiquement, au contact des lymphocytes, certaines cellules basales évoluent vers la nécrose. Comme dans le lichen plan idiopathique, il existe une hypergranulose, une hyperkératose orthokératosique et un infiltrat dense du derme

Tableau 3 : Similitudes et différences cliniques entre le lichen plan commun et le lichen dans la GVHDc Clinical similarities and differences between common oral planus lichen and lichen in GVHDc

\begin{tabular}{|c|c|c|}
\hline & Lichen plan idiopathique & Lichen plan dans la GVHDc \\
\hline Aspect clinique & $\begin{array}{l}\text { Lésions blanches réticulées }(+++) \text {, } \\
\text { en plaques, papuleuses, verruqueuses } \\
\text { (rares) } \\
\text { érythémateux, érosif, atrophique }\end{array}$ & $\begin{array}{l}\text { Lésions blanches en plaques, verruqueuses }(+++) \text {, } \\
\text { peu de formes réticulées } \\
\text { Atrophie majeure, érosions diffuses, } \\
\text { érosions torpides }\end{array}$ \\
\hline Sièges & $\begin{array}{l}\text { Joues ( } 1 / 3 \text { post. face int.) } \\
\text { Face dorsale langue } \\
\text { Gencives }\end{array}$ & $\begin{array}{l}\text { Joues ( } 1 / 3 \text { post. face int.) } \\
\text { Face dorsale langue } \\
\text { Gencives }\end{array}$ \\
\hline Evolution & $\begin{array}{l}\text { Par poussées avec périodes } \\
\text { de rémission (LP quiescent) } \\
\text { Transformation maligne sur lichen } \\
\text { plan érosif et atrophique : } 1 \text { à } 5 \%\end{array}$ & $\begin{array}{l}\text { Atrophie diffuse } \\
\text { Lésions blanches stables, érosions torpides } \\
\text { Transformation maligne plus élevée }\end{array}$ \\
\hline
\end{tabular}


qui « érode » la couche basale épidermique. En 1981, Janin et coll. [8] réalisaient la description princeps de l'aspect ultrastructural du lichen dans la GVHDc et le comparaient avec celle du lichen plan idiopathique. Les lésions lichéniennes apparaissent vers le $38^{\mathrm{e}}$ jour de la GVHD. Elles ressemblent à celles du lichen plan idiopathique : atteinte des cellules basales, nombre et répartition des corps colloïdes, présence de cellules régénératives. Des lymphocytes « satellites » des kératinocytes lésés sont présents dans ces lésions lichéniennes. De plus le nombre important des mastocytes dans l'infiltrat inflammatoire laisse présager des remaniements conjonctifs caractéristiques de la phase scléreuse tardive de la GVHD. Les mastocytes présents dans les lésions lichéniennes anciennes et dans les scléroses débutantes sont responsables de la genèse de la fibrose $[8,9]$. II a été démontré in vitro que la tryptase mastocytaire était capable de stimuler la production de collagène. De même, les lymphocytes $\mathrm{T}$, et plus particulièrement les lymphokines, induisent la synthèse de collagène in vitro.

Le lichen plan associé à la GVHD se présente souvent sous une forme clinique et sémiologique particulière, difficile à traiter. La prise en charge de la pathologie buccale des patients atteints d'une GVHD consiste à traiter le lichen plan dans la mesure où il répond aux traitements et à prévenir l'évolution maligne assez fréquente. Plusieurs traitements ont été proposés mais seuls les corticoïdes, les rétinoïdes et la PUVA ont une effica- cité bien documentée. Le thalidomide agirait en intervenant à un stade précoce de reconnaissance antigénique et de l'activation des lymphocytes $T$ du donneur, en inhibant la réponse lymphocytaire normale. II est essentiellement efficace dans la GVHDc, peu ou pas dans la GVHDa. Au total, il a une action modulatrice complexe sur le système immunitaire. II est utilisé à fortes doses (800 à 1600 mg/j) dans les GVHDc résistantes au traitement immunosuppresseur ou à haut risque de mortalité. Ce n'est pas un traitement anodin, il existe de nombreux effets secondaires dont les neuropathies périphériques et aucune étude contrôlée n'a prouvée son efficacité.

Les meilleures réponses thérapeutiques utilisant la corticothérapie entraînent une amélioration dans 20 à $60 \%$ des cas et elle se manifeste après 6 à 8 semaines ; à l'arrêt du traitement, la rechute est possible, le plus souvent après arrêt brutal. Le tacrolimus en traitement topique est une bonne alternative thérapeutique pour les lichens érosifs rebelles à la corticothérapie mais c'est un traitement relativement coûteux.

Le développement des greffes allogéniques de moelle osseuse constitue un réel progrès thérapeutique car il a permis la rémission de pathologies jadis fatales. La GVHDc reste une complication majeure avec un risque également à long terme par le développement secondaire d'un carcinome épidermoïde dont la localisation buccale est fréquente (Fig. 14 a et b). Le traitement de la GVHDc entraîne une immunodépres-
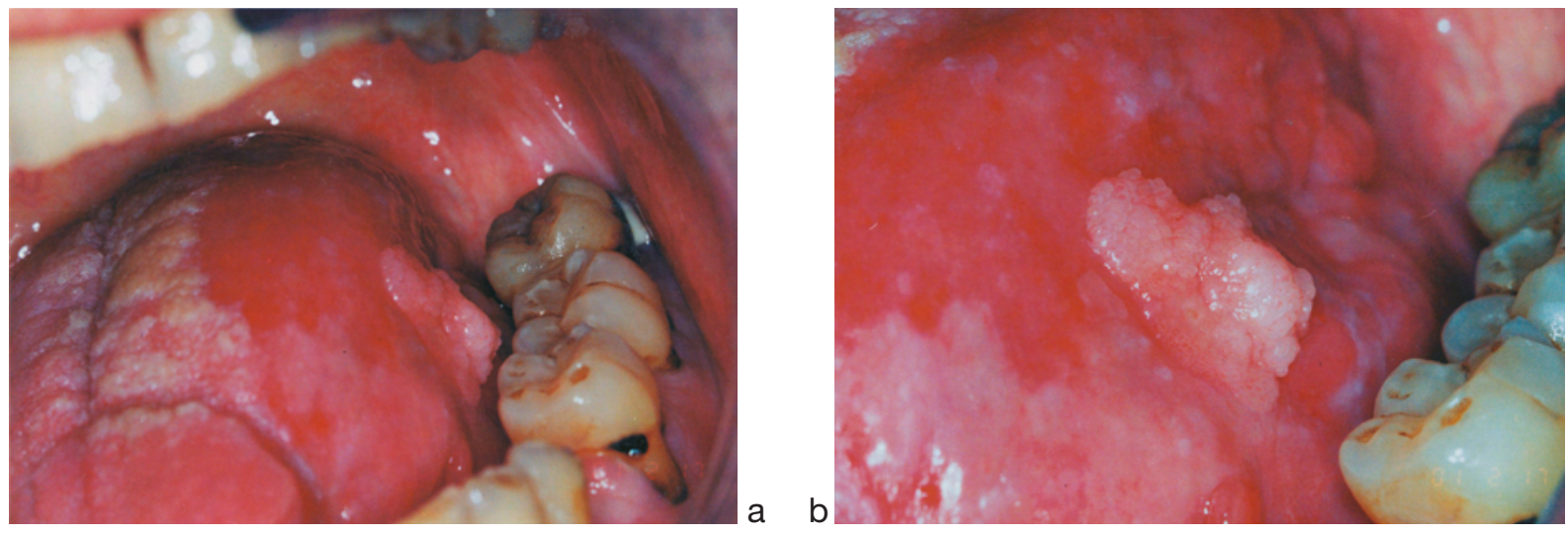

Figure 14 : $(a$ et b) : Carcinome épidermoïde de forme bourgeonnante se développant sur un lichen érosif torpide dû à une GVHD.

Squamous cell carcinoma of budding form developing on a torpid erosive lichen due to a GVHD. 
médecine

buccale

chirurgie

buccale

VOL. $12, \mathrm{~N}^{\circ} 1$ 2006

page 54 sion chronique qui peut favoriser le développement de cancers secondaires. II est connu et rapporté depuis longtemps que l'immunosuppression est associée à une grande variété de tumeurs (lymphomes, tumeurs cutanées...). Le développement de tumeurs solides est moins commun ; la littérature montre ainsi que les patients traités pour une maladie de Hodgkin ont un risque élevé de cancérisation secondaire surtout après un délai de 5 à 10 ans. Les tumeurs solides retrouvées sont variables. Le cancer secondaire retrouvé le plus fréquemment chez les patients atteints de GVHDc est le carcinome épidermoïde. Le traitement combinant radiothérapie et chimiothérapie ne fait qu'augmenter le risque [10]. Les hématologues recommandent la limitation de la radiothérapie et la suppression de l'imurel dans le traitement de la GVHD car ce sont des inducteurs de tumeurs secondaires. La présence d'un lichen plan dans la GVHDc et son évolution torpide constituent un risque accru de développement de carcinome épidermoïde, en moyenne huit ans après la greffe [11]. II se développe préférentiellement sur des lésions lichéniennes érosives ou atrophiques torpides. Ces tumeurs ont une extension intra-épithéliale très importante. Cette caractéristique impose des exérèses larges, souvent impossibles car trop mutilantes.

Les premières observations de cancer secondaire remontent à la fin des années 1980. En 1984, Deeg et coll. rapportent vingt cas de cancers secondaires à une greffe de moelle osseuse (19 cas traités pour une affection hématologique, un pour aplasie médullaire) : 17 d'entre eux ont reçu dans leur conditionnement une irradiation corporelle totale (8 à 15,75 Gy). Ces cancers secondaires appartiennent à trois groupes. Le premier groupe comprend six rechutes leucémiques survenues entre les 62 et 1074 jours après la greffe ; le deuxième groupe un lymphome apparu 54 à 730 jours après la greffe ; le troisième des cancers solides apparus 347 à 1875 jours après la greffe. Pour les auteurs, ces cancers secondaires ont une origine multifactorielle associant l'irradiation corporelle totale, l'immunosuppression prolongée et la présence du virus Epstein Barr [24].

En 1988, Howe et coll. ont rapporté le premier cas de carcinome épidermoïde dans une GVHDc car ils pensaient qu'il ne s'agissait pas d'une association fortuite [12].

En 1989, Witherspoon et coll. ont essayé de déterminer l'incidence de cancers secondaires après greffe allo, syn ou autogénique pour le traitement des leucémies $(n=1926)$ et pour le traitement de l'aplasie médullaire $(n=320)$ : 35 ont présenté une pathologie tumorale secondaire dont 16 LNH, 6 leucémies, 13 cancers solides (glioblastomes, mélanomes, carcinomes épidermoïdes,...). Ils concluent que pendant la première année post-greffe, il existe un risque de 1,2\% de cancers secondaires [13].

Lishner et coll. en 1990 ont publié une étude sur les tumeurs cutanéo-muqueuses survenant après greffe de moelle osseuse. 56 patients ont été suivis à long terme (5 ans) : 10 présentaient comme pathologie initiale une anémie sévère ou aplasie, 16 une LAM, 11 une LLA et 19 une LMC. Le conditionnement pré-greffe comportait une chimiothérapie et une irradiation corporelle totale ; 3 ont développé un cancer cutané ou buccal (deux carcinomes épidermoïdes, un mélanome malin). Ces 3 patients avaient une GVHDc et ont été traités par immunosuppresseurs pendant une période prolongée [14]. Flowers et coll. en 1992 rapportent une étude portant sur 17 greffes de moelle osseuse chez des malades atteints d'une anémie de Fanconi traités entre 1973 et 1990. Les donneurs de moelle étaient HLA géno-identiques pour $n=14$, phéno-identiques pour $n=2$ et un mal assorti. Le conditionnement associait cyclophosphamide, busulfan et 12 Gy d'irradiation corporelle totale. Sur les 12 patients, 7 ont survécu entre 1 et 17 ans après la greffe de moelle osseuse. La probabilité de survie à 5 ans est estimée à $65 \%$; 2 ont développé un carcinome épidermoïde localisé sur la langue dix ans après la greffe [7]. Quinonez et coll. en 1993 développent l'idée de la présence de corps intracytoplasmiques dans les carcinomes épidermoïdes buccaux à partir d'un patient normal atteint de carcinome épidermoïde et deux patients atteints de GVHDc, et présentant tous deux un carcinome épidermoïde buccal. La présence de corps intracytoplasmiques est considérée comme une caractéristique diagnostique de l'adénocarcinome et n'a pas été rapporté [15]. Altman et coll. en 1994 rap- 
portent un cas clinique de développement d'un carcinome épidermoïde pendant une PUVAthérapie pour le traitement d'une GVHDc [16]. Hoffman et coll. en 1994 développent l'impact de la radiothérapie au niveau de la toxicité mucocutanée et dans l'apparition de cancers secondaires chez les patients atteints de GVHDc [23]. En 1997, Otsubo et coll. rapportent un cas de carcinome épidermoïde gingival chez une patiente de 21 ans ayant eu une allogreffe de moelle osseuse cinq ans auparavant pour le traitement d'une anémie sévère [17].

Zhang et coll. en 2002 confirment que les carcinomes épidermoïdes buccaux sont en augmentation chez les patients allogreffés. Ils ont étudié et comparé des carcinomes buccaux avec cinq carcinomes épidermoïdes de patients allogreffés. Ils ont retrouvé plus d'ADN de HPV dans les carcinomes épidermoïdes développés chez les sujets allogreffés (3 cas, $60 \%$ des cas) que dans les autres carcinomes épidermoïdes (4 cas sur 34 soit $12 \%$ des cas) (18). Harima et coll. ont également montré en 2002 l'importance des HPV dans la survenue des carcinomes épidermoïdes après radiothérapie. La présence des HPV a été analysée par PCR (réaction en chaîne par polymérase) et basée sur les séquences consensus E6 et L1. Ils sont mis en évidence des HPV dans $76,2 \%$ des cas et la présence des HPV influence de façon significative la survie des sujets ayant des cancers [19].

\section{CONCLUSION}

L'importance des manifestations buccales dans la GVHDa et la GVHDc est comparable à celles des autres tissus cibles, mais la spécificité de la cavité buccale nécessite une attention particulière. En effet la cavité buccale, première étape de la digestion, participe au goût, à la mastication, à la déglutition et facilite l'élocution. La disparition de ces fonctions engendre des répercussions à tout niveau. La vie quotidienne est perturbée avec une gêne pour parler, masti- quer, avaler ; un inconfort parfois très pénible en résulte.

Ce travail montre donc le rôle primordial des spécialistes s'intéressant à la région bucco-faciale (odontologistes, stomatologistes, dermatologues, $\mathrm{ORL}$ ) dans le diagnostic, la prévention et le traitement de ces complications bucco-dentaires. Un suivi régulier pré, per et post-greffe permet au patient de mieux appréhender ses pathologies muqueuse, parodontale et odontologique [20]. Les lésions lichéniennes, d'évolution parfois fulminante, et l'atrophie des glandes salivaires constituent les atteintes les plus fréquentes [21]. Une mise en état de la cavité buccale, pour éliminer tous les facteurs irritatifs et infectieux, doit être réalisée systématiquement avant la greffe. Une prise en charge adéquate de l'état buccal permet de réduire les effets secondaires de l'hyposialie, de suivre l'évolution des lésions lichénoïdes et de dépister l'apparition de lésions tardives [21].

Un examen buccal systématique avec une analyse spécifique par biopsie muqueuse permettra d'évaluer le statut et l'évolution d'une GVHDc [22]. Dans la GVHD, les dysplasies de la muqueuse buccale constituent un indicateur important de risque de transformation maligne et ne doivent pas être considérées comme un changement réactif aux mucites.

Toutes ces données mettent en exergue l'importance d'un suivi régulier (tous les quatre mois) des lésions, même quiescentes, chez des patients après greffe de moelle osseuse. Les lésions lichéniennes doivent donc être régulièrement contrôlées et biopsiées car la transformation maligne de ces lésions se fait tardivement après greffe de moelle allogénique, au cours de la longue période de GVHDc. Une biopsie de toutes les lésions torpides, blanches ou érosives, doit être systématique. La découverte d'un carcinome impose une chirurgie carcinologique qui semble peu efficace à moyen terme. La radiothérapie est fortement déconseillée car elle représente un facteur aggravant. Le pronostic reste sombre, le décès survenant un à deux ans après la découverte du premier carcinome.

\author{
médecine \\ buccale \\ chirurgie \\ buccale \\ VOL. $12, \mathrm{~N}^{\circ} 1$ \\ 2006 \\ page 55
}




\section{RÉFÉRENCES}

1 - BARRETT AP, Bilous AM. Oral patterns of acute and chronic graft-versus-host disease. Arch Dermatol 1984; 120 : 1461-5.

2 - Kolbinson DA, Schubert MM, Flournoy N, Truelove EL. Early oral changes following bone marrow transplantation. Oral Surg Oral Med Oral Pathol 1988; 66 : 130-8.

3 - JOHNSON ML, FARMER ER. Graft versus host reactions in dermatology. J Am Acad Dermatol 1998; 38 : 369-92 (quiz 393-6).

4 - GluCKMan E, Janin-Mercier A. La réaction du greffon contre l'hôte. Les maladies systémiques. Kahn MarcelFrancis, Pettier André-Paul, Meyer Olivier, Piette Jean Charles. Editions Médecine-sciences Flammarion, Paris, 3 édition 1991.

5 - YASHER S, HAN KF, HALEY JC. Lichen sclerosus lichen planus overlap in a patient with hepatitis $\mathrm{C}$ virus infection. Br J Dermatol 2004; 150 :168.

6 - Carcassi C, Cottoni F, Floris L, La Nasa G, Mulargia M, Baldini G, Senes G, Orru S, Montesu MA, Cerimele D. The HLA-DRB1*0101 allele is responsible for HLA susceptibility to lichen ruber planus. Eur J Immunogenet $1994 ; 21: 425-9$.

7 - Flowers ME, Doney KC, Storb R, EeEg HJ, Sanders Je, Sullivan KM, Bryant E, Witherssoon RP, Appelbaum FR, BuCKNeR CD ET AL. Marrow transplantation for Fanconi anemia with or without leukemic transformation: an update of the Seattle experience. Bone Marrow Transplant $1992 ; 9: 167-73$.

8 - Janin-Mercier A, Aaurat JH, Bourges M, Sohier J, DidieRJEAN L, GLuCKMAN E. Ultrastructure de la phase lichénienne de la réaction chronique du greffon contre I'hôte. Similitudes avec le lichen plan idiopathique. Ann Dermatol Venereol $1981 ; 108$ : 49-56.

9 - Claman HN, Holda JH, MaieR T, CHOI KL.Chronic graftvs.-host disease: changes in natural suppressor cells and mast cells. Isr J Med Sci 1988 ; 24 : 367- 71.

10 - Atree SV, Crilley PA, Conroy JF, Mically B, Brodsky JT. Cancer of the esophagus folowing allogeneic bone marrow transplantation for acute leukemia. Am J Clin Oncol $1995 ; 18: 343-7$

11 - Socie G, Stone JV, Wingard JR, Weisdorf D, HensleeDOWNeY PJ, BRedeson C, CAHN JY, PASSWEG JR, Rowlings PA, Schouten HC, KolB HJ, KLeIN JP. Longterm survival and late deaths after allogeneic bone marrow transplantation. Late Effects Working Committee of the International Bone Marrow Transplant Registry. N Engl J Med 1999 ; $341: 14-21$

12 - HowE NR, LANG PG. Squamous cell carcinoma of the sole in a patient with chronic graft-versus-host disease. Arch Dermatol 1988 ; 124 : 1244-5.

13 - Witherspoon RP, Fisher LD, Schoch G, Martin P, SULLIVAN KM, SANDERS J. Secondary cancers after bone marrow transplantation for leukemia or aplastic anemia. N Eng J Med 1989 ; 321 : 784-9.
14 - Lishner M, Patterson B, Kandel R, Fyles G, Curtis Je, MEHARCHAND J. Cutaneous and mucosal neoplasms in bone marrow transplant recipients. Cancer 1990 ; 65 : 473-6.

15 - QUINONEZ G, AHING S, DIOCEE M. Intracytoplasmic lumina in squamous epithelial lesions of the oral cavity. Pathol Res Pract 1993 ; 189 : 293-7.

16 - Altman JS, AdLER SS. Development of multiple cutaneous squamous cell carcinomas during PUVA treatment for chronic graft-versus-host disease. J Am Acad Dermatol $1994 ; 31$ : 505-7.

17 - Otsubo H, Yokoe H, Miya T, Atsuta F, Miura N, TANZAWA $H$. Gingival squamous cell carcinoma in a patient with chronic graft-versus-host-disease. Oral Surg Oral Med Oral Pathol 1997 ; 84 : 171-4.

18 - Zhang L, EPSTEIN JB, POH CF, BeREAN K, LAM WL, ZHANG Z, RosIn MP. Comparaison of HPV infection, P53 mutation and allelic losses in post transplant and non posttransplant oral squamous cell carcinomas. J Oral Pathol Med 2002 ; 31 : 134-41.

19 - Harima Y, SaWAda S, Nagata K, SOUGaWa M, OHnishi T. Human papilloma virus (HPV) DNA associated with prognosis of cervical cancer after radiotherapy. Int J Radiat Oncol Biol Phys 2002 ; 52 : 1345-51.

20 - Franca CM, Domingues-Martins M, Volpe I, Filho RS, SoARES dE ARAujo N. Severe oral manifestations of chronic graft-vs-host disease. J Am Dent Assoc 2001; 132 : 1124-7.

21 - Samson J, Nedir R, Helg C, Bischof M. Manifestations stomatologiques de la GVHD et leurs traitements. Acta Med Dent Helv 1999; 4 : 181-94.

22 - NaKAMURA S, HIROKI A, ShinOHARA M, Gondo H, OHYAMa Y, Mouri T, SasakI M, ShIRAsuna K, HaRada M, $\mathrm{N}$ IHO Y. Oral involvement in chronic graft-versus-host disease after allogeneic bone marrow transplantation. Oral Surg Oral Med Oral Pathol 1996; 82 : 556-63.

23 - Hoffman W, Rodemann HP, Schmidberger H, Weiss B, BAMBERG M. Enhanced muco-cutaneous toxicity of radiotherapy in a patient with chronic GVHD and secondary malignancy. Ann Oncol 1994; 5 : 377-8.

24 - Deeg HJ, Sanders J, Martin P, Fefer A, Neiman P, Singer J, Storb R, Thomas ED. Secondary malignancies after marrow transplantation. Exp Hematol 1984; 12 : 660-6.

NDLR. Depuis la soumission de cet article, un article sur ce sujet est paru dans une revue francophone :

Fricain JC, Sibaud V, Hafian H, Deminiere C, Taieb A, BOIRON JM. Les manifestations buccales de la réaction du greffon contre l'hôte. Ann Dermatol Venereol 2005 ; 132 : 1017-25. 